\title{
Surgical Suture Assembled with Tadalafil/Polycaprolactone Drug-Delivery for Vascular Stimulation Around Wound: Validated in a Preclinical Model
}

\author{
Reza Sayyad Soufdoost ${ }^{1}$ (D), Seyed Ali Mosaddad ${ }^{2}$ (D), Yalda Salari ${ }^{3}$ (D), Mohsen Yazdanian 4,* ${ }^{(D)}$, Hamid \\ Tebyanian $^{4, *(D)}$, Elahe Tahmasebi 4 (D), Alireza Yazdanian 5 (D), Ali Karami ${ }^{4}$ (D), Aref Barkhordari ${ }^{6}$ (D) \\ 1 Dentistry Research Institute, School of Dentistry, Shahed University, Tehran, Iran \\ 2 Student Research Committee, School of Dentistry, Shiraz University of Medical Sciences, Shiraz, Iran \\ 3 Department of Oral and Maxillofacial Radiology, Faculty of Dentistry, Tehran Medical Sciences, Islamic Azad University, \\ Tehran, Iran \\ 4 Research Center for Prevention of Oral and Dental Diseases, Baqiyatallah University of Medical Sciences, Tehran, Iran. \\ 5 Department of veterinary, Science and Research Branch, Islamic Azad University, Tehran, Iran \\ 6 Division of Toxicology, Department of Comparative Biosciences, Faculty of Veterinary Medicine, University of Tehran, \\ Tehran, Iran \\ * Correspondence: drmyazdanian@yahoo.com (M.Y); tebyan.hamid@yahoo.com (H.T);
}

Scopus Author ID 57198792244 (M.Y); 55220019400 (H.T)

Received: 14.04.2020; Revised: 7.05.2020; Accepted: 9.05.2020; Published: 13.05.2020

\begin{abstract}
In this study, a novel Polycaprolactone suture assembled with Tadalafil was investigated to improve wound healing processes via vascular stimulation. Tadalafil/Polycaprolactone (TP) suture was developed by the electrospinning method. The designed suture was characterized by SEM, mechanical properties assessments, tensile strength measurements and the drug release study. For in vivo tests, rats were classified into two study groups. An incision was made on their back skin and they were sutured with TP suture and Polycaprolactone suture as control. Rats were sacrificed at 7 days following surgery for histopathological examinations with Hematoxylin and Eosin staining. Results of Tensile test demonstrated that the lowest tensile strength belonged to 3 and $4 \%$ wt and the highest tensile strength belonged to 1 and $2 \%$ wt of TP suture. The rate of Tadalafil release showed that the highest drug release was related to 3 and $4 \%$ wt which were about 125 to $210 \mu \mathrm{g}$ during 15 days. The histopathology revealed that the number of blood vessels, collagen fibers, fibroblast, polymorphonuclear leukocytes, and epithelization was remarkable in Tadalafil/Polycaprolactone group during 7-day. A novel Tadalafil/Polycaprolactone suture improved the processes of wound healing by releasing the Tadalafil drug around the sutured wound and can be used in medical applications.
\end{abstract}

Keywords: Suture; Wound; Tadalafil; Polycaprolactone.

(C) 2020 by the authors. This article is an open access article distributed under the terms and conditions of the Creative Commons Attribution (CC BY) license (https://creativecommons.org/licenses/by/4.0/).

\section{Introduction}

Sutures are surgical threads used to repair surgical cuts by a strand of natural or synthetic origins [1]. To select suture material and manage wounds, depth of suture placement, the number of tissue layers, suture removal time, tension across the wound, presence of oedema, adequate strength, and inflammatory reactions play a pivotal role [2]. To better perform suturing, strands should be pliable and flexible. They should also be easy to tie knot, knots should be highly secure, and materials should be free from irritating or infectious 
substances [3]. Highly flexible sutures should be employed to manage surgical sutures $[4,5]$. Commercially available suture materials can meet most, but not all the requirements. The wound healing process after surgery is almost disrupted due to surrounding inflammations in spite of histocompatible materials and minimally invasive procedures newly introduced; in other words, surgical research is focused on the optimization of the tissue healing process. Different factors contribute to the healing process; i.e. underlying diseases, old age, poor nutrition, immune deficiencies, and sepsis. Hence, the stimulation of healing process is the main target of current studies [6-9].

Recent advances in medicine introduced new sutures with significant advantages in order to customize and improve suturing outcomes; for example, antimicrobial sutures [10], bioactive sutures containing DNA [11, 12], drugs [13, 14], antibodies [15], proteins [16], growth factors [17], or silver [18]. In spite of the attractiveness of the topic, commercially available products with such properties are very few $[19,20]$. Such systems facilitate the delivery of the substance at the surgical site, which ensures biological activity due to a sufficient level of the drug. to minimize immune reactions, toxicity, and side effects, the carrier materials should be biologically absorbable and compatible; they should not cause local inflammations or physical blocking due to incomplete metabolism since it may suppress the healing process $[5,21]$. They have to be made of sterilizable and flexible components in order to facilitate substance delivery. The substances should not be absorbed before exerting the effects; hence, they have to be chosen from constant release drugs [4]. Postoperative complications including infection of the surgical site can be controlled and wound healing process can be accelerated by using drug-eluting sutures depending on the drug used [22].

Using drug-eluting sutures obviates the need for supplemental drugs, in which systematic administrations reduce their potency or availability at the surgical site. Utilization of constant release drugs at the surgical site sustains concentration for a prolonged time, while there is no toxic effect on the systemic circulation [2]. The employment of antimicrobial sutures in some procedures reduces the risk of infection and is cost-effective [19, 20, 23]. Since this therapeutic approach seems very promising, researchers try to fabricate sutures loaded with antithrombotic, analgesic, antineoplastic and anti-proliferative agents [1]. Drug-eluting sutures should maintain the drug concentration at the desired level and preserve the mechanical properties, while using degrading polymers and controlling drug release strategies [2]. Different procedures including dip method to coat the surface [24], grafting [25], and electrospinning are used to develop the sutures [1]. An electrostatic spinning, a versatile polymer processing technique, a polymer solution or melt is exposed to a high electric field, which in turn forms nano-dimension fibers that form a nonwoven fabric, which is applicable to a number of purposes. electrospinning is easy to use, highly efficient, cost-effective, and highly reproducible [26, 27]. Polycaprolactone, a semi-crystalline, aliphatic, slow biodegradable, high biocompatible, and good drug permeable polymer [28].

Polycaprolactone, unlike poly(D,L-lactic-co-glycolic acid) commonly used as a biodegradable polymer, cannot produce acidic environments due to degradation [29]. Hence, Polycaprolactone can be considered as a promising biomedical polymer due to its good mechanical properties and lower cost. Polycaprolactone products called electrospun fibers and co-polymers can be employed in a variety of spinning solutions and conditions. Casalini et al., in a study made a bioresorbable suture loaded with lidocaine and evaluated the release rate in tissue in a theoretical model. They reported that drug-eluting potency of the suture was improved by increasing the diffusion coefficient and the drug half-life [30]. In comparison with 
Polycaprolactone fibers loaded with diclofenac, the release rate and mechanical features of the melt spun bioactive sutures modified with diclofenac hydrotalcite nano-hybrid were much better [1]. Tadalafil is an advantageous inhibitor of phosphodiesterase enzyme type-5 (PDE5), currently used to treat pulmonary arterial hypertension and erectile dysfunction. Breakdown of nitric oxide-driven cGMP in vascular smooth muscle cells and platelets is prohibited by inhibiting PDE-5 using Tadalafil; it vasodilates peripheral arteries in smooth muscles and inhibits adhesion and aggregation of platelets [31]. Tadalafil protects myocardium, lung, brain and skin flap, against ischemia [32-35]. Therefore, the current study aimed to evaluate the vascular stimulation of a newly produced Polycaprolactone/Tadalafil suture to accelerate the healing process around the wound.

\section{Materials and Methods}

\subsection{Materials.}

In the current study, Tadalafil and Polycaprolactone were obtained from SigmaAldrich. The methanol, chloroform and solvents were purchased from Carlo Erba (Italy) and Sigma-Aldrich (USA).

\subsection{Preparation of drug-delivery sutures.}

Polycaprolactone, chloroform, methanol, and Tadalafil were used to produce suture. Firstly, Polycaprolactone and Tadalafil powder with a solvent were mixed on hot plate with a stirring to prepare a homogeneous solution for 1 hour. Polycaprolactone and Tadalafil were dissolved in methanol and chloroform, respectively. Therefore, the volume ratio of methanol to chloroform (1:9) was used for achieving the best homogeneous solution. The concentration of Polycaprolactone and Tadalafil was 14 and 1-4 gr in a $100 \mathrm{ml}$ solution, respectively. Next step, the prepared solution was fed into a syringe of the electrospinning apparatus. The rotating speed of the collector was set at $1500 \mathrm{rpm}$ and the distance between the needle tip to the drum surface was fixed at $8 \mathrm{~cm}$. After electrospinning, the samples were grounded under the conditions of 9 kilovolt and $0.3 \mathrm{ml} / \mathrm{h}$ feed rate to form fiber bundles and they were kept to evaporate the solvent completely for $24 \mathrm{~h}$. Finally, the fibers were twisted into yarns using hand-driven twist tester (Alfred Suter Co. Inc) (Figure 1A) [36].

\subsection{Tensile Strength Measurement.}

A DMA 6800 (TA Instruments, Timonium, MD) was employed to determine the medicinal properties of the suture. samples were vertically clamped in $3-\mathrm{cm}$ long and a 5newton $(\mathrm{N})$ load cell was applied at $0.05 \mathrm{~N} / \mathrm{min}$ to stretch the sample until tearing.

\subsection{In vitro drug release study.}

For this purpose, 4-cm in length drug-delivery sutures were immersed with continuous agitation in $2 \mathrm{~mL}$ PBS (pH 7.4) at $37^{\circ} \mathrm{C}$. Then, $1 \mathrm{~mL}$ of the aliquot was replaced with $1 \mathrm{~mL}$ PBS and then the absorbance rate was read at $264 \mathrm{~nm}$ by a spectrophotometer (UV-1800, Shimadzu, Japan). 

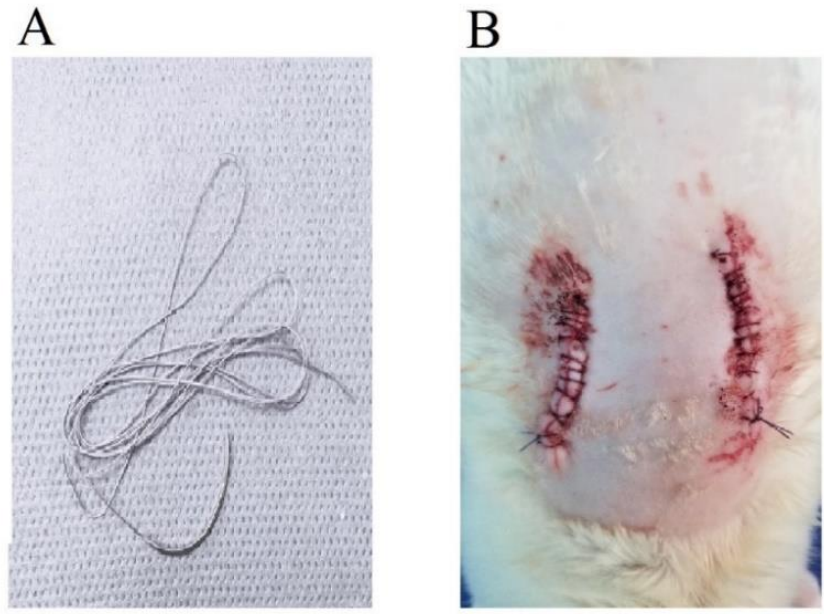

Figure 1. A: Fabricated Tadalafil/Polycaprolactone suture, B: in vivo test with Tadalafil/Polycaprolactone and Polycaprolactone sutures.

\subsection{In vivo vascularization evaluation.}

The study protocol for experiments and animal caring was approved by the Ethical Committee for Animal Research of Baqiyatallah University of Medical Sciences, Tehran, Iran. Wistar male rats (weigh 200-250 g) purchased from Pasteur Institute, Tehran, Iran. Rats were kept in stainless steel cages at $22^{\circ} \mathrm{C}$ with $12: 12 \mathrm{~h}$ light/dark cycle. For acclimation, the animals were kept at their cages for one week with free access to standard rodent chow pellets. Animals were assigned to two experiment groups ( $n=6$ in each group) based on time as seven days. To induce anesthesia, ketamine/xylazine $(90 / 10 \mathrm{mg} / \mathrm{kg})$ was used, and then, middle back of rat was opened with two incisions ( $4 \mathrm{~cm}$ length) and followed with shaving the site and scrubbing with Povidone-iodine. Tadalafil/Polycaprolactone (TP) and Polycaprolactone (P) suture (control group) were used in the current study and one incision was stitched with TP suture and another incision was stitched with P suture. Rats were sacrificed after seven days from surgery and the samples were prepared for next step (Figure 1B).

\subsection{Histological examination.}

After surgery, the rats were euthanized to obtain the suture surrounding tissue and examine the biocompatibility of sutures. The tissue was rinsed in saline and fixed in paraformaldehyde (10\%). And then, samples were embedded in paraffin and sectioned into 5$\mu \mathrm{m}$ slices using microtome. And then, the sections were stained with Hematoxylin and Eosin staining. Finally, sections were observed by a light Microscope (Leica Microsystems, Wetzlar, Germany). Some important factors which can affect the healing process such as reepithelization, PMNL (Polymorphonuclear leucocytes), fibroblasts, new vessels, and new collagen was evaluated by semi-quantitative method in this study. Sections were evaluated according to the scale: $0,1,2,3,4$ (Table 1) by two independent observers [37].

\subsection{Statistics.}

Mean values \pm SEM were calculated for each parameter and data from the semiquantitative evaluation were analyzed using non-parametric Kruskall-Wallis test. The level of significance was set at $\mathrm{p}<0.05$. 
Table 1. Explanation of used scale in the semi-quantitative evaluation of histological sections (ST - surrounding tissue, i.e. tissue out of GT; DL - demarcation line; SCT - subcutaneous tissue; GT - granulation tissue).

\begin{tabular}{c|c|c|c|c|c} 
Scale & Epithelization & PMNL & Fibroblasts & New Vessels & Collagen \\
\hline 0 & Thickness of cut edges & Absent & Absent & Absent & Absent \\
\hline 1 & Migration of cells $(<50 \%)$ & Mild-ST & Mild-ST & Mild-SCT & Minimal-GT \\
\hline 2 & Migration of cells $(>50 \%)$ & Mild-DL/GT & Mild-GT & Mild-GT & Mild-GT \\
\hline 3 & Bridging of excision & Moderate DL/GT & Moderate-GT & Moderate-GT & Moderate-GT \\
\hline 4 & keratinization & Marked-DL/GT & Marked-GT & Marked-GT & Marked-GT
\end{tabular}

\section{Results and Discussion}

\subsection{Tensile strength and in vitro drug release results.}

Tensile test results of melt-spun filaments are shown in figure 2A. The PT suture 1 and $2 \% \mathrm{wt}$ had the highest tensile strength about 1.03 and $1.02 \mathrm{~N}$, respectively. This amount of tensile strength was reduced with increasing of Tadalafil concentration to $4 \% \mathrm{wt}$. The lowest tensile strength belonged to 3 and $4 \%$ wt of PT suture approximately 0.98 and $0.96 \mathrm{~N}$, respectively. The rate of Tadalafil release from suture was measured in media and in different concentrations of chloride and phosphate to determine drug release. The complete release of Tadalafil from the suture was ensured by PBS in order to measure its release on fibers. In all concentrations of Tadalafil, the drug release was gradually increased during 15 days. The Tadalafil 1 and $2 \%$ wt showed that the lowest drug release was 50 and $75 \mu \mathrm{g}$ to near 110 and $140 \mu \mathrm{g}$ during 15 days. The highest drug release was related to 3 and $4 \%$ wt which were about 125 to $210 \mu \mathrm{g}$ during 15 days (Figure 2B). Tadalafil/Polycaprolactone 4\% wt was selected for the next step according to obtained results.

A

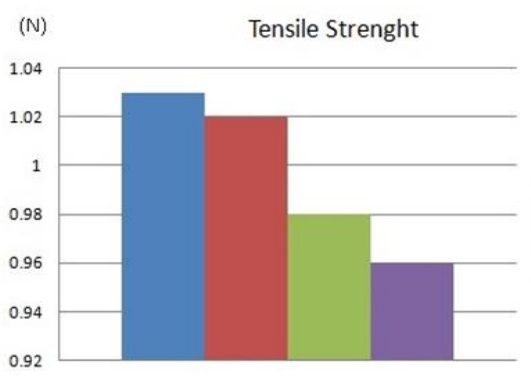

B

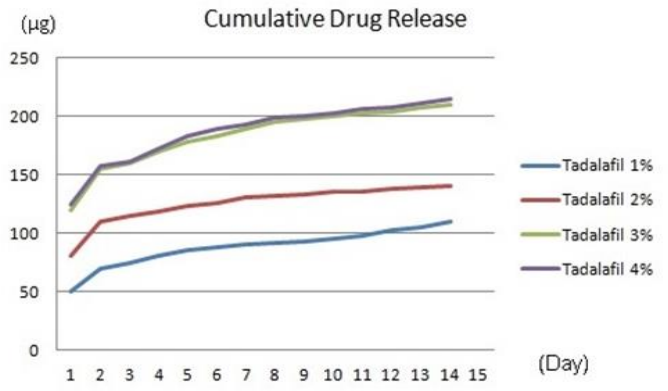

Figure 2. A: Tensile strength, B: in vitro drug release of Tadalafil/Polycaprolactone and Polycaprolactone groups.

\subsection{Histopathology results.}

The microscopic results revealed that the blood vessels were increased in Tadalafil/Polycaprolactone 4\% wt suture in comparison with Polycaprolactone around the sutured wound. In addition, the number of collagen fibers and fibroblasts in the Tadalafil/Polycaprolactone group was more than the Polycaprolactone group after 7 days (Figure 3). Polymorphonuclear leukocytes (PMNL) were considerably increased in the Tadalafil/Polycaprolactone group in comparison with the Polycaprolactone group. Figure 4 shows the significant migration of cells around Tadalafil/Polycaprolactone suture and this amount was considerable and more than the Polycaprolactone group. 


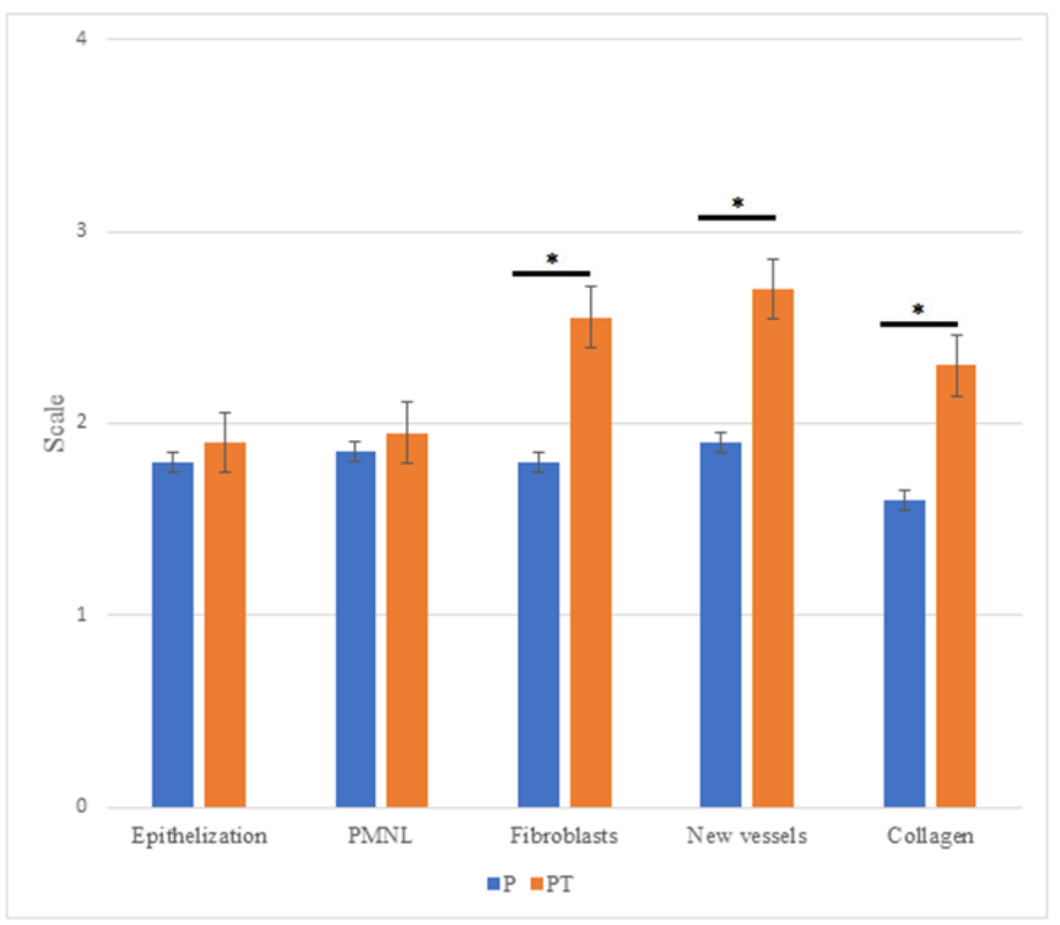

Figure 3. Semi-quantitative analysis of histological after surgery ( 7 days, $\left.{ }^{*} \mathrm{p}<0.05\right)$.
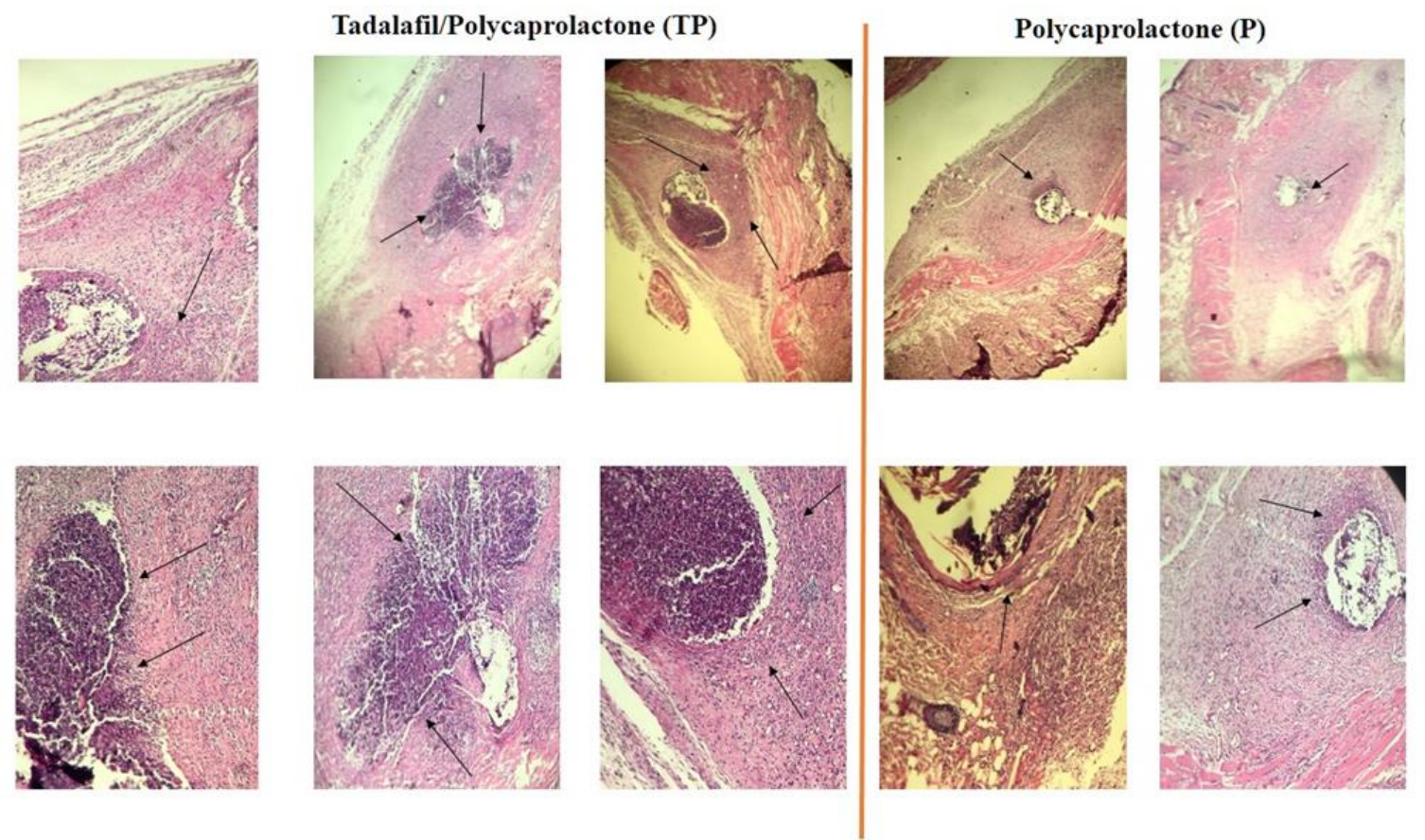

Figure 4. Hematoxylin and Eosin staining of Tadalafil/Polycaprolactone and Polycaprolactone groups (magnification, $\times 40$ and 100).

\subsection{Discussion.}

It is still essential to select a suitable suture for a patient. In this regard, any allergic reactions of soft tissue to the suture material should be considered [21]. Sutures are surgical threads used to repair surgical cuts by a strand of natural or synthetic origins. Although staples, tapes, and glues are the commercially available devices to clinically manage cuts, sutures are the most common ones with an annual market of 1.3 billion US\$ [38]. However, no ideal product that can meet all the requirements is available thus far and they are mostly selected in 
surgeries based on availability and familiarity [39]. A suture should be mechanically appropriate, have a good resorption rate and low risk of infection and inflammation. To better fulfill such requirements, new materials are developed in recent years. Development of medicated sutures containing a bioactive compound with constant release profile in order to better help tissue repair is currently the goal of many studies [1]; in this regard, much attention is attracted to drug-eluting sutures for different clinical purposes [21]. These sutures, in addition to closing the cuts, can prevent complications and even play a therapeutic role [30]. The new generation of sutures has additional advantages of local constant drug release [40]. However, poor tensile strength, constant drug release, and scale of commercial viability are the main obstacles that limit the development of drug-eluting sutures [21, 40-42]. These sutures can easily be used in surgeries since they are very thin [43]. About 12 million ocular procedures are performed annually worldwide that use nylon sutures to close wounds and incisions [44].

In this study, a novel Polycaprolactone suture was assembled with Tadalafil to improve wound healing processes via vascular stimulation. The development of a suture loaded with Tadalafil and absorbable Polycaprolactone was described in the current study. A drug-delivery suture that could alleviate the liver pain during wound healing was introduced in an animalmodel study by Lee et al. They reported that the suture could constantly control the pain, in addition to its biocompatible and mechanical properties. the PLC sheet was also used in the suture to deliver ibuprofen in order to alleviate the pain and the results showed that it could control the pain similar to oral administration of the drug, but with lower doses and good invivo biocompatibility [21]. An animal-model study by Fuchs et al. used sutures coated with insulin-like growth factor-I for local delivery. Monofilament sutures were used to create anastomoses of the colon in animals. They reported that the growth factor was locally delivered to the site using sutures coated with bioabsorbable poly ( $\mathrm{D}$, L-lactide) suspension. Hence, the wound was locally managed with no need to expose the whole organism to the damaging doses [4]. In a rat hernia model study by Korenkov et al., TGF- $\beta 1$ was applied into the abdominal wall to increase the strength, but the results were not affirmative [45]. To investigate postsurgical pain control, the efficacy of a suture coated with sedatives was assessed in a study by Mahantesha et al. A total of 20 patients undergoing flap surgery received phase I therapy. They reported that the patients' pain level reduced using sedative-coated suture that serves as a local drug delivery system, and minimizes the side effects of conventional systemic drug exposure [5]. Liu et al. in an in-vitro study evaluated the antimicrobial properties of the Yarns with established diameter and crystallinity, and the release rate from the Yarns that contained ampicillin in different doses. The results showed by adding ampicillin into the polymer, the diameter of the fiber reduced and lower diameter variations were observed. The inhibition zone test results of the Yarns against Staphylococcus aureus and Klebsiella pneumonia indicated a constant release rate of ampicillin after the production and antimicrobial activity of the as-spun Yarns. They reported that the e-spun nanofiber Yarns can potentially be used as biomaterials for surgical purposes to decrease the infection rate in the site [36].

An antibiotic-eluting suture developed by Kashiwabuchi et al. was used for ophthalmic surgery. Poly (L-lactide), polyethylene glycol, and levofloxacin were employed to produce the suture by wet electrospinning. Histological analyses were performed on sections of surrounding sutures tissue implanted into Sprague-Dawley rat corneas to investigate the biocompatibility of the suture. The results showed that the suture could successfully deliver the drug locally with constant release rate; in addition, wet electrospinning provided a suitable platform to meet size requirements for ophthalmic surgeries; however, tensile strength should 
be improved [43]. A medicated suture was developed by a novel method in the study by Lee et al., in which a polymeric sheet loaded with a sedative was covered on a common suture. The suture has mechanical advantages with a six-day drug-release profile. To design a bioactive suture and optimize its effects, it is of great importance to control its drug release rate [21]. Catanzano et al. used Polycaprolactone in their study to develop a composite bioactive suture with anti-inflammatory effects that were loaded with diclofenac (Dic) or one of its nanohybrids while the drug was intercalated in a synthetic hydrotalcite (HT-Dic). The melt-spinning method was employed to prepare the fibers at different POLYCAPROLACTONE /HTDic/Dic ratios. According to their results, adding Dic or HT-Dic could significantly affect the fibers tensile strength; the knots, however, had a low effect on the mechanical behavior of the suture. But Dic-loaded fibers could significantly exert anti-inflammatory effects in-vivo [1].

In this study, the lowest tensile strength belonged to 3 and $4 \% \mathrm{wt}$ of PT suture approximately 0.98 and $0.96 \mathrm{~N}$, respectively. This amount of Tadalafil could decrease the strength of suture and on the other side, the highest drug release belonged to these concentrations of Tadalafil and could release about 125 to $210 \mu \mathrm{g}$ during 15 days. Tadalafil/Polycaprolactone 4\% wt was selected for in vivo test in rats. Histopathological results showed that the Tadalafil could increase the number of blood vessels around the sutured wound and also, the remarkable migration of cells around suture was detected in Tadalafil/Polycaprolactone group. Collagen fibers and epithelization were considerable in the Tadalafil/Polycaprolactone group in comparison to the Polycaprolactone group due to the proliferation of blood vessels around the sutured wound after seven days.

\section{Conclusions}

An appropriate suture must have some prperties such as mechanically, resorption rate and low risk of infection and inflammation. Development of sutures contains a bioactive compound with constant release profile in order to help tissue repair. This research demonstrated a assembled novel Polycaprolactone suture with Tadalafil to improve wound healing processes via vascular stimulation. the lowest tensile strength and highest drug release (about 125 to $210 \mu \mathrm{g}, 15$-day) related to 3 and $4 \%$ wt. Tadalafil/Polycaprolactone $4 \%$ wt was selected for the next step according to obtained results. The number of blood vessels, collagen fibers, fibroblast, polymorphonuclear leukocytes, and epithelization was remarkable in Tadalafil/Polycaprolactone suture group. Utilization of constant release drugs at the surgical site sustains concentration for a prolonged time, while there is no toxic effect on the systemic circulation. It can be concluded that Tadalafil/Polycaprolactone suture could accelerate the wound healing process by releasing the Tadalafil drug during 7 days. This suture can be considered as a suitable product for medical applications due to the simplicity of its fabrication and being a cost-effective product.

\section{Funding}

This research received no external funding.

\section{Acknowledgments}

The authors would like to Research Center for Prevention of Oral and Dental Diseases, Baqiyatallah University of Medical Sciences, Tehran, Iran for their kind help. 


\section{Conflicts of Interest}

\section{The authors declare no conflict of interest.}

\section{References}

1. Catanzano, O.; Acierno, S.; Russo, P.; Cervasio, M.; Del Basso De Caro, M.; Bolognese, A.; Sammartino, G.; Califano, L.; Marenzi, G.; Calignano, A.; Acierno, D.; Quaglia, F. Melt-spun bioactive sutures containing nanohybrids for local delivery of anti-inflammatory drugs. Mater Sci Eng C Mater Biol Appl 2014, 43, 3009, https://doi.org/10.1016/j.msec.2014.07.012.

2. Dennis, C.; Sethu, S.; Nayak, S.; Mohan, L.; Morsi, Y.Y.; Manivasagam, G. Suture materials - Current and emerging trends. J Biomed Mater Res A 2016, 104, 1544-59, https://doi.org/10.1002/jbm.a.35683.

3. Hochberg, J.; Meyer, K.M.; Marion, M.D. Suture choice and other methods of skin closure. Surg Clin North Am 2009, 89, 627-41, https://doi.org/10.1016/j.suc.2009.03.001.

4. Fuchs, T.F.; Surke, C.; Stange, R.; Quandte, S.; Wildemann, B.; Raschke, M.J.; Schmidmaier, G. Local delivery of growth factors using coated suture material. ScientificWorldJournal 2012, 2012, https://doi.org/10.1100/2012/109216.

5. Mahantesha, S.; Ashwini, S.; Manjusha, M.V.; Ramya, G.; Shivani, S.R.D. Comparative Evaluation of the Efficacy of Surgical Suture Coated with Analgesic versus Conventional Suture for Sustained Local Pain Relief- Pilot Study. J Chem Pharm Rese 2018, 10, 13-21.

6. Fuchs, T.F.; Surke, C.; Stange, R.; Quandte, S.; Wildemann, B.; Raschke, M.J.; Schmidmaier, G. Local delivery of growth factors using coated suture material. TheScientificWorldJournal 2012, 2012, 109216109216, https://doi.org/10.1100/2012/109216.

7. Wu, J.; Landen, N.X. Investigation of Skin Wound Healing Using a Mouse Model. Methods Mol Biol 2020, 2154, 239-247, https://doi.org/10.1007/978-1-0716-0648-3_20.

8. Damkerngsuntorn, W.; Rerknimitr, P.; Panchaprateep, R.; Tangkijngamvong, N.; Kumtornrut, C.; Kerr, S.J.; Asawanonda, P.; Tantisira, M.H.; Khemawoot, P. The Effects of a Standardized Extract of Centella asiatica on Postlaser Resurfacing Wound Healing on the Face: A Split-Face, Double-Blind, Randomized, PlaceboControlled Trial. J Altern Complement Med 2020, https://doi.org/10.1089/acm.2019.0325.

9. Salas-Oropeza, J.; Jimenez-Estrada, M.; Perez-Torres, A.; Castell-Rodriguez, A.E.; Becerril-Millan, R.; Rodriguez-Monroy, M.A.; Canales-Martinez, M.M. Wound Healing Activity of the Essential Oil of Bursera morelensis, in Mice. Molecules 2020, 25, https://doi.org/10.3390/molecules25081795.

10. Leaper, D.; McBain, A.J.; Kramer, A.; Assadian, O.; Sanchez, J.L.; Lumio, J.; Kiernan, M. Healthcare associated infection: novel strategies and antimicrobial implants to prevent surgical site infection. Ann $R$ Coll Surg Engl 2010, 92, 453-8, https://doi.org/10.1308/003588410x12699663905276.

11. Kim, H.; Kim, B.H.; Huh, B.K.; Yoo, Y.C.; Heo, C.Y.; Choy, Y.B.; Park, J.H. Surgical suture releasing macrophage-targeted drug-loaded nanoparticles for an enhanced anti-inflammatory effect. Biomater Sci 2017, 5, 1670-1677, https://doi.org/10.1039/c7bm00345e.

12. Li, Y.; Xu, T.; Tu, Z.; Dai, W.; Xue, Y.; Tang, C.; Gao, W.; Mao, C.; Lei, B.; Lin, C. Bioactive antibacterial silica-based nanocomposites hydrogel scaffolds with high angiogenesis for promoting diabetic wound healing and skin repair. Theranostics 2020, 10, 4929-4943, https://doi.org/10.7150/thno.41839.

13. Kim, H.; Kim, B.H.; Huh, B.K.; Yoo, Y.C.; Heo, C.Y.; Choy, Y.B.; Park, J.H. Surgical suture releasing macrophage-targeted drug-loaded nanoparticles for an enhanced anti-inflammatory effect. Biomater Sci 2017, 5, 1670-1677, https://doi.org/10.1039/c7bm00345e.

14. de Souza, M.L.; Dos Santos, W.M.; de Sousa, A.; de Albuquerque Wanderley Sales, V.; Nobrega, F.P.; de Oliveira, M.V.G.; Neto, P.J.R. Lipid Nanoparticles As A Skin Wound Healing Drug Delivery System: Discoveries And Advances. Curr Pharm Des 2020, https://doi.org/10.2174/1381612826666200417144530.

15. Shibuya, T.Y.; Wei, W.Z.; Zormeier, M.; Ensley, J.; Sakr, W.; Mathog, R.H.; Meleca, R.J.; Yoo, G.; June, C.H.; Levine, B.; Lum, L.G. Anti-CD3/anti-CD28 monoclonal antibody-coated suture enhances the immune response of patients with head and neck squamous cell carcinoma. Arch. Otolaryngol. Head Neck Surg 1999, 125, 1229-34, https://doi.org/10.1001/archotol.125.11.1229.

16. Horvathy, D.B.; Vacz, G.; Cselenyak, A.; Weszl, M.; Kiss, L.; Lacza, Z. Albumin-coated bioactive suture for cell transplantation. Surg Innov 2013, 20, 249-55, https://doi.org/10.1177/1553350612451353.

17. Cummings, S.H.; Grande, D.A.; Hee, C.K.; Kestler, H.K.; Roden, C.M.; Shah, N.V.; Razzano, P.; Dines, D.M.; Chahine, N.O.; Dines, J.S. Effect of recombinant human platelet-derived growth factor-BB-coated sutures on Achilles tendon healing in a rat model: A histological and biomechanical study. J Tissue Eng 2012, 3, https://doi.org/10.1177/2041731412453577.

18. Blaker, J.J.; Nazhat, S.N.; Boccaccini, A.R. Development and characterisation of silver-doped bioactive glass-coated sutures for tissue engineering and wound healing applications. Biomaterials 2004, 25, 1319-29, https://doi.org/10.1016/j.biomaterials.2003.08.007.

19. Ming, X.; Nichols, M.; Rothenburger, S. In vivo antibacterial efficacy of MONOCRYL plus antibacterial suture (Poliglecaprone 25 with triclosan). Surg Infect (Larchmt) 2007, 8, 209-14, https://doi.org/10.1089/sur.2006.004. 
20. Ming, X.; Rothenburger, S.; Nichols, M.M. In vivo and in vitro antibacterial efficacy of PDS plus (polidioxanone with triclosan) suture. Surg Infect (Larchmt) 2008, 9, 451-7, https://doi.org/10.1089/sur.2007.061.

21. Lee, J.E.; Park, S.; Park, M.; Kim, M.H.; Park, C.G.; Lee, S.H.; Choi, S.Y.; Kim, B.H.; Park, H.J.; Park, J.H.; Heo, C.Y.; Choy, Y.B. Surgical suture assembled with polymeric drug-delivery sheet for sustained, local pain relief. Acta Biomater 2013, 9, 8318-27, https://doi.org/10.1016/j.actbio.2013.06.003.

22. Chen, X.; Hou, D.; Wang, L.; Zhang, Q.; Zou, J.; Sun, G. Antibacterial Surgical Silk Sutures Using a HighPerformance Slow-Release Carrier Coating System. ACS Appl Mater Interfaces 2015, 7, 22394-403, https://doi.org/10.1021/acsami.5b06239.

23. Rothenburger, S.; Spangler, D.; Bhende, S.; Burkley, D. In vitro antimicrobial evaluation of Coated VICRYL* Plus Antibacterial Suture (coated polyglactin 910 with triclosan) using zone of inhibition assays. Surg Infect (Larchmt) 2002, 3, 79-87, https://doi.org/10.1089/sur.2002.3.s1-79.

24. Wang, L.; Chen, D.; Sun, J. Layer-by-layer deposition of polymeric microgel films on surgical sutures for loading and release of ibuprofen. Langmuir 2009, 25, 7990-4, https://doi.org/10.1021/la9004664.

25. Gupta, B.; Jain, R.; Singh, H. Preparation of antimicrobial sutures by preirradiation grafting onto polypropylene monofilament. Polym advan technol 2008, 19, 1698-1703, https://doi.org/10.1002/pat.1146.

26. Shi, X.; Zhou, W.; Ma, D.; Ma, Q.; Bridges, D.; Ma, Y.; Hu, A. Electrospinning of Nanofibers and Their Applications for Energy Devices. J. Nanomater 2015, 2015, 1-20, https://doi.org/10.1155/2015/140716.

27. Ahmed, J.; Gultekinoglu, M.; Edirisinghe, M. Bacterial cellulose micro-nano fibres for wound healing applications. Biotechnol Adv 2020, https://doi.org/10.1016/j.biotechadv.2020.107549.

28. Li, Y.; Liao, C.; Tjong, S.C. Synthetic Biodegradable Aliphatic Polyester Nanocomposites Reinforced with Nanohydroxyapatite and/or Graphene Oxide for Bone Tissue Engineering Applications. Nanomaterials (Basel, Switzerland) 2019, 9, 590, https://doi.org/10.3390/nano9040590.

29. Woodard, L.N.; Grunlan, M.A. Hydrolytic Degradation and Erosion of Polyester Biomaterials. ACS macro letters 2018, 7, 976-982, https://doi.org/10.1021/acsmacrolett.8b00424.

30. Casalini, T.; Masi, M.; Perale, G. Drug eluting sutures: a model for in vivo estimations. Int J Pharm 2012 , 429, 148-57, https://doi.org/10.1016/j.ijpharm.2012.03.024.

31. Li, Z.; Xi, X.; Gu, M.; Feil, R.; Ye, R.D.; Eigenthaler, M.; Hofmann, F.; Du, X. A stimulatory role for cGMPdependent protein kinase in platelet activation. Cell 2003, 112, 77-86, https://doi.org/10.1016/s00928674(02)01254-0.

32. Rao, Y.J.; Xi, L. Pivotal effects of phosphodiesterase inhibitors on myocyte contractility and viability in normal and ischemic hearts. Acta Pharmacol Sin 2009, 30, 1-24, https://doi.org/10.1038/aps.2008.1.

33. Tsai, B.M.; Turrentine, M.W.; Sheridan, B.C.; Wang, M.; Fiore, A.C.; Brown, J.W.; Meldrum, D.R. Differential effects of phosphodiesterase-5 inhibitors on hypoxic pulmonary vasoconstriction and pulmonary artery cytokine expression. Ann Thorac Surg 2006, 81, 272-8, https://doi.org/10.1016/j.athoracsur.2005.06.040.

34. Ko, I.G.; Shin, M.S.; Kim, B.K.; Kim, S.E.; Sung, Y.H.; Kim, T.S.; Shin, M.C.; Cho, H.J.; Kim, S.C.; Kim, S.H.; Kim, K.H.; Shin, D.H.; Kim, C.J. Tadalafil improves short-term memory by suppressing ischemiainduced apoptosis of hippocampal neuronal cells in gerbils. Pharmacol Biochem Behav 2009, 91, 629-35, https://doi.org/10.1016/j.pbb.2008.10.009.

35. Oh, M.; Chang, H.; Minn, K.W. The effects of tadalafil on axial-pattern skin flap survival in rats. Dermatol Surg 2008, 34, 626-30, https://doi.org/10.1111/j.1524-4725.2007.34118.x.

36. Liu, H.; Leonas, K.K.; Zhao, Y. Antimicrobial Properties and Release Profile of Ampicillin from Electrospun Poly (E-caprolactone) Nanofiber Yarns. J Eng Fiber Fabr 2010, 5, 10-19, https://doi.org/10.1177/155892501000500402.

37. Gal, P.; Kilik, R.; Mokry, M.; Vidinsky, B.; Vasilenko, T.; Mozes, S.; Bobrov, N.; Tomori, Z.; Bober, J.; Lenhardt, L. Simple method of open skin wound healing model in corticosteroid-treated and diabetic rats: standardization of semi-quantitative and quantitative histological assessments. Vet Med 2008, 53, 652-9, https://doi.org/10.17221/1973-VETMED.

38. Pillai, C.K.; Sharma, C.P. Review paper: absorbable polymeric surgical sutures: chemistry, production, properties, biodegradability, and performance. J Biomater Appl 2010, 25, 291-366, https://doi.org/10.1177/0885328210384890.

39. Moy, R.L.; Waldman, B.; Hein, D.W. A review of sutures and suturing techniques. J Dermatol Surg Oncol 1992, 18, 785-95, https://doi.org/10.1111/j.1524-4725.1992.tb03036.x.

40. Weldon, C.B.; Tsui, J.H.; Shankarappa, S.A.; Nguyen, V.T.; Ma, M.; Anderson, D.G.; Kohane, D.S. Electrospun drug-eluting sutures for local anesthesia. J Control Release 2012, 161, 903-9, https://doi.org/10.1016/j.jconrel.2012.05.021.

41. Choudhury, A.J.; Gogoi, D.; Chutia, J.; Kandimalla, R.; Kalita, S.; Kotoky, J.; Chaudhari, Y.B.; Khan, M.R.; Kalita, K. Controlled antibiotic-releasing Antheraea assama silk fibroin suture for infection prevention and fast wound healing. Surgery 2016, 159, 539-47, https://doi.org/10.1016/j.surg.2015.07.022.

42. Joseph, J.; Nair, S.V.; Menon, D. Integrating Substrateless Electrospinning with Textile Technology for Creating Biodegradable Three-Dimensional Structures. Nano Letters 2015, 15, 5420-5426, https://doi.org/10.1021/acs.nanolett.5b01815. 
43. Kashiwabuchi, F.; Parikh, K.S.; Omiadze, R.; Zhang, S.; Luo, L.; Patel, H.V.; Xu, Q.; Ensign, L.M.; Mao, H.Q.; Hanes, J.; McDonnell, P.J. Development of Absorbable, Antibiotic-Eluting Sutures for Ophthalmic Surgery. Transl Vis Sci Technol 2017, 6.

44. Grinstaff, M.W. Designing hydrogel adhesives for corneal wound repair. Biomaterials 2007, 28, 5205-5214, https://doi.org/10.1016/j.biomaterials.2007.08.041.

45. Korenkov, M.; Yuecel, N.; Koebke, J.; Schierholz, J.; Morsczeck, C.; Tasci, I.; Neugebauer, E.A.M.; Nagelschmidt, M. Local administration of TGF- $\beta 1$ to reinforce the anterior abdominal wall in a rat model of incisional hernia. Hernia 2005, 9, 252-258, https://doi.org/10.1007/s10029-005-0341-y. 